\title{
Lifting the lockdown: what are the options for low and middle-income countries?
}

Rajiv Chowdhury*1, PhD; Shammi Luhar*1, PhD; Nusrat Khan*1, MSc; Sohel Reza Choudhury², $\mathrm{PhD}$; Imran Matin ${ }^{3}, \mathrm{PhD}$; and Oscar $\mathrm{H} \mathrm{Franco}^{4}$, PhD.

1. Department of Public Health and Primary Care, University of Cambridge, Cambridge, United Kingdom.

2. National Heart Foundation and Research Institute, Dhaka, Bangladesh.

3. BRAC Institute for Global Development, Dhaka, Bangladesh.

4. Institute for Preventive and Social Medicine, University of Bern, Bern, Switzerland.

*Contributed equally.

Word count: 1985; One Figure.

Correspondence to: Dr Shammi Luhar

Department of Public Health and Primary Care

University of Cambridge

Strangeways Research Laboratory

Cambridge, CB1 8RN, United Kingdom

Email: s1989@medschl.cam.ac.uk 


\section{ABSTRACT}

To limit the social, economic and psychological damage caused by strict social distancing interventions, many low and middle-income countries (LMICs) are seeking to ease restrictions. However, it is unknown what a 'safe reopening' entails in LMICs given suboptimal diagnostic and surveillance capabilities. Here we discuss three community-based public health measures (sustained mitigation, zonal lockdown and dynamic rolling lockdowns) which seek to adequately balance the public health and economic priorities. Each of these options have limitations and prerequisites that may be context-specific and should be considered before implementation, including implementation and maintenance costs, social and economic costs, context-specific epidemic growth and the existing health resources.

\section{INTRODUCTION}

The coronavirus disease-2019 (COVID-19) pandemic has claimed more than 400,000 lives worldwide[1] and has been responsible for significant economic disruptions globally[2]. Similar to the high-income nations, low and middle-income countries (LMICs) also responded to COVID19 by implementing various population-level measures, including strict nationwide lockdowns and physical distancing[3]. Worldwide, with no effective treatments for COVID-19 and a vaccine at least a year away, these measures have been effective in preventing health systems from becoming overloaded, especially in the LMICs where: 1) the risk of disease transmission is high (populations are often large and dense, with a high degree of interaction and physical contact), 2) awareness of how to prevent disease is often poor (eg, clean water and hygiene practices), 3) public health systems are often under-resourced (eg, safety equipment and intensive care units/ICU), and 4) access to healthcare is limited and reliant on largely out-of-pocket payment.

These strict social distancing interventions come with a price: they are unsustainable in the long term given their social, economic and psychological impacts. For example, a recently completed survey in Bangladesh showed that after its initial days of lockdown, a staggering $72 \%$ of urban and $54 \%$ of rural households had lost their main source of earnings[4]. Therefore, many LMICs are currently lifting the lockdowns, irrespective of the status of infection and the level of contagion. It remains, however, unclear what would be an optimal strategy for 'safe re-opening' (given the likelihood of disease resurgence), especially across low-income settings, where diagnostic capacities and surveillance infrastructure is poor[5].

In this regard, we have considered three community-based public health strategies for LMICs, which aim to strike a balance between health protection and preventing economic collapse, and discuss possible application, ideal pre-requisites, and inherent limitations for each. They include: 
1) sustained mitigation, 2) zonal lockdown, 3) rolling lockdown (dynamic measures). These strategies (Figure 1) are not mutually exclusive and could be further adapted and combined depending on local needs and disease progression.

\section{SUSTAINED MITIGATION}

Following the primary lockdown, staying on a 'mitigation-only' phase (a strategy adopted by developed countries such as France, Switzerland and Italy) has involved measures such as physical distancing, wearing masks, test-trace-isolation of positive cases, shielding of the vulnerable and banning mass gatherings[6]. The successful implementation of this approach, however, is contingent on a number of key factors.

First, the early implementation of a strict lockdown has resulted in a significant reduction of contact rates and has reduced new infections[7],[8]. By contrast, many LMICs, where lockdown has recently been lifted, appear to have an upward trend of cases and deaths[9]. Second, availability of high levels of surveillance, mass testing and rapid case isolation are essential to avoid the potential for resurgent epidemics, and facilitate containment[10],[11]. Third, for contact-tracing, enough trained contact tracers (or scalable digital platforms) should be available, with a relatively sparse target population (minimising the possibility of super-spreading events). The effectiveness of contact-tracing might be importantly minimised in large, dense countries such as Bangladesh ( 1300 people/sqkm), compared to sparsely populated countries like Spain ( $\sim 90$ people/sqkm)[12]. Contact-tracing is also less effective at the height of community spread when the rates are on the rise. Fourth, individual and population-level adherence to mitigation measures (eg, physical distancing, hygiene, home quarantine) must be adequate. For many LMICs, this remains a challenge given large-scale social stigma and suboptimal risk communication strategies[13]. Finally, healthcare services must be able to adequately cope with the resurgence in new cases, including availability of specialised care, hospitals and ICU beds. In many LMICs, there is however a chronic shortage of 1 ) critical care infrastructure (only 48000 ventilators are available in India to serve its 1.3 billion people[14]), 2) personal protective equipment (PPE), 3) training of health workforce, and 4) good working conditions - all of which reduce system efficiency and enhance likelihood of transmission among healthcare workers.

Despite being less restrictive than full lockdown, a mitigation-only strategy is not immune to financial hardship as it can also lead to socioeconomic disruption - somewhat compromising its sustainability over a prolonged period. For example, Sweden, which adopted some of the most liberal mitigation measures such as keeping restaurants, bars, and gyms open throughout the previous few months, whilst encouraging physical distancing rules, is expected to suffer $\sim 10 \%$ contraction in its economy in 2020 according to the Swedish Central Bank[15]. 


\section{ZONAL LOCKDOWN}

The idea of fencing between infected and healthy communities, termed cordon sanitaire, has been deployed during a variety of outbreaks for centuries. In line with this principle, as an exit strategy, many countries have transitioned to a system of "zonal lockdown"[16]. This system entails identification of specific "hotspots" where a sudden outbreak cluster, with a high number of cases, have been identified in real time. Such clustered social distancing works by dividing the population into "zones" according to the geospatial distribution of disease cluster contained within, so that interactions within a zone are significantly greater than interactions between zones[17]. Transmission hotspots, or "red zones" are subject to strict lockdown measures than "green zones", where very few or no new cases have been identified for several days. Such strategies were adopted in France[17], with green zones defined by areas where the virus transmission is relatively low and there is not as burdensome pressure placed on the healthcare system.

The "zonal lockdown" approach has several important requirements. First, this categorisation of hotspots is typically a dynamic process, which requires an ability to reliably identify, in real time, areas that meet or fall short of the pre-specified lockdown criteria. This requires continuous datadriven feedbacks on: 1) regional daily confirmed cases (either by date of reporting or onset of symptoms), and 2) other time-series information needed to calculate the changes in regionspecific effective reproduction number $(R$, the average number of secondary infections per infected individual), including daily numbers of hospitalized cases, daily numbers of deaths in different age groups, and transmission dynamics (eg, average time from infection to death) [17]. While such strategy has been successfully established in developed settings (such as France, where testing is widespread with 0.52 daily tests being done per 1000 population), this remains challenging in many LMICs due to 1) absence of large-scale population surveillance system based on randomly-selected individuals (e.g., in Bangladesh, the testing approach has focused on purposive, self-referred samples, with significant selection bias), and 2) poor testing laboratory facilities and reporting capacities (e.g., in Pakistan, only 0.09 daily tests are being conducted per 1000 individuals)[18]. In this regard, India has adapted a scalable mass "Pool testing" approach[19]. This cost-effective strategy involves collecting multiple samples in a tube and testing them with a single RT-PCR assay run. If the test is negative, all the people tested are negative. If it is positive, every person has to be tested individually for the virus. This approach reduces the time needed to test large swathes of the population[20].

Second, the classification of the zones should also be multifactorial. This should not only take into consideration the incidence rate, but also the other epidemiological (e.g., doubling rate of new cases; number of deaths) and administrative aspects (e.g., available hospital and ICU beds; testing and surveillance structure; residential versus industrial zone). Third, managing the zones efficiently to reduce transmission both within and outside of the zones is a major undertaking. 
Recent reports from India shows that infection size in many containment areas is 100 -fold to 200 fold higher than the cases reported at those sites - indicating that containment efforts within zones may not have fully paid off[21]. Therefore, detailed apriori standard operating procedures should be devised to include aspects on 1) within-zone public health measures (eg, risk communication, house-to-house surveillance, test booths, contact-tracing, case referral systems, ambulance and medical facilities), 2) within-zone measures of emergency services (eg, food supply, law enforcement, isolation centres, and burial facilities), and 3) outside-zone measures such as creation of "buffer" zones (e.g., in India[19]) that surround the main containment zone to minimise out-of-zone transmissions. Such detailed protocols are crucial for efficiency. In Iran, for example, suboptimal zone management has increased risk of a second wave[22]. Finally, similar to sustained mitigation strategy, the zonal lockdown will be most effective when the overall rate of infection is in decline, accompanied by exhaustive vigilance.

While zonal lockdown, if implemented properly, can help contain the spread of the virus, efficacy of this approach can be reduced by other concurrent transmission networks, such as those linked to economic and social interdependency between zones[17]. Additionally, the impacts on the economy, particularly inside the zones, can be considerably more severe than under mitigation where the economy essentially opens with restrictions, exacerbating economic hardship in countries with already weak economic performance and social security nets. Therefore, these aspects merit careful consideration during the planning phase of this strategy.

\section{ROLLING LOCKDOWN}

Dynamic or "rolling" lockdown measures take place when strict social distancing measures are applied and lifted periodically. This strategy has been described as a robust measure to minimise uncertainty in both effective $R$ values, and in the severity of the virus (i.e. the proportion of cases requiring ICU admission)[23]. This approach may be potentially suitable for LMICs with large and dense populations, high patterns of contact, poor economic/health systems resilience, and weak testing/contact tracing capacities. Furthermore, this approach aims to provide a balance between avoiding health systems being overloaded and grinding economies completely to a halt[24]. A recent paper mathematically modelled the effects of either a strict 50-day suppression or a 50day mitigation, followed by 30 days of relaxation (during which businesses are allowed to reopen, with basic hygiene measure kept in place), in 16 economically diverse countries. A strict 50-day lockdown, that reduces the effective $R$ value to 0.5 , can prevent health systems overload and leads to considerably fewer deaths (130,000 during 18 months in the 16 countries they modelled) compared to a more relaxed 50-day mitigation/30-day relaxation cycle ( $\sim 3.5$ million predicted deaths globally) and under no-intervention (counter-factual) scenario ( 8 million predicted deaths)[25]. To further contextualize, a subsequent paper estimated that 1) a single, one-off 
lockdown will be insufficient to bring the pandemic under control, and 2) secondary peaks could be larger than the first, without continued restrictions[26].

However, as with the other strategies, this approach is also contingent on several factors. First, before implementing a rolling lockdown, every developing country should carefully consider the economic and social costs to implement these measures. Second, impacts on incidence and casefatality will rely on local levels of adequate adherence to social distancing measures. Third, this approach would also bring a new set of logistical challenges. Therefore, countries will need to formulate bespoke plans for reorganising business supply chains, so that they align with the economy opening and closing. While such readjustments to complement a schedule of lockdown is not ideal, unprecedented challenges often require unusual and adaptive solutions, especially if other alternative exit strategies are not feasible. Furthermore, by establishing a detailed surveillance system while the lockdown takes place, countries can modify the duration of the lockdown and relaxation periods according to the behaviour of the epidemic in their settings. A recent example of this has been in Pakistan, where the World Health Organization has recommended a 14-day-on/14-day-off rolling lockdown to control the epidemic[27].

In conclusion, several strategies exist to enable the LMICs to safely return to 'normality', allowing for preservation of health with controlled economic implications. Beyond the impact that the virus has on health and mortality, the measures taken to counteract its effect have relevant implications for the financial infrastructure of a nation and the mental health of its citizens. It is essential to consider these potential options to identify balanced alternatives to tackle the pandemic. However, each option discussed here has important prerequisites and limitations, and their impacts may vary across settings. Therefore, a suitable, "context-specific" strategy should be based on several inter-related considerations: 1) local epidemic growth rate, 2) existing health infrastructure (to survey, test, and treat, at scale), 3) social and economic costs, and 4) carefullydevised plans to implement and sustain the measures. 
Figure 1: Summary of various post-lockdown community-based public health strategies for LMICS

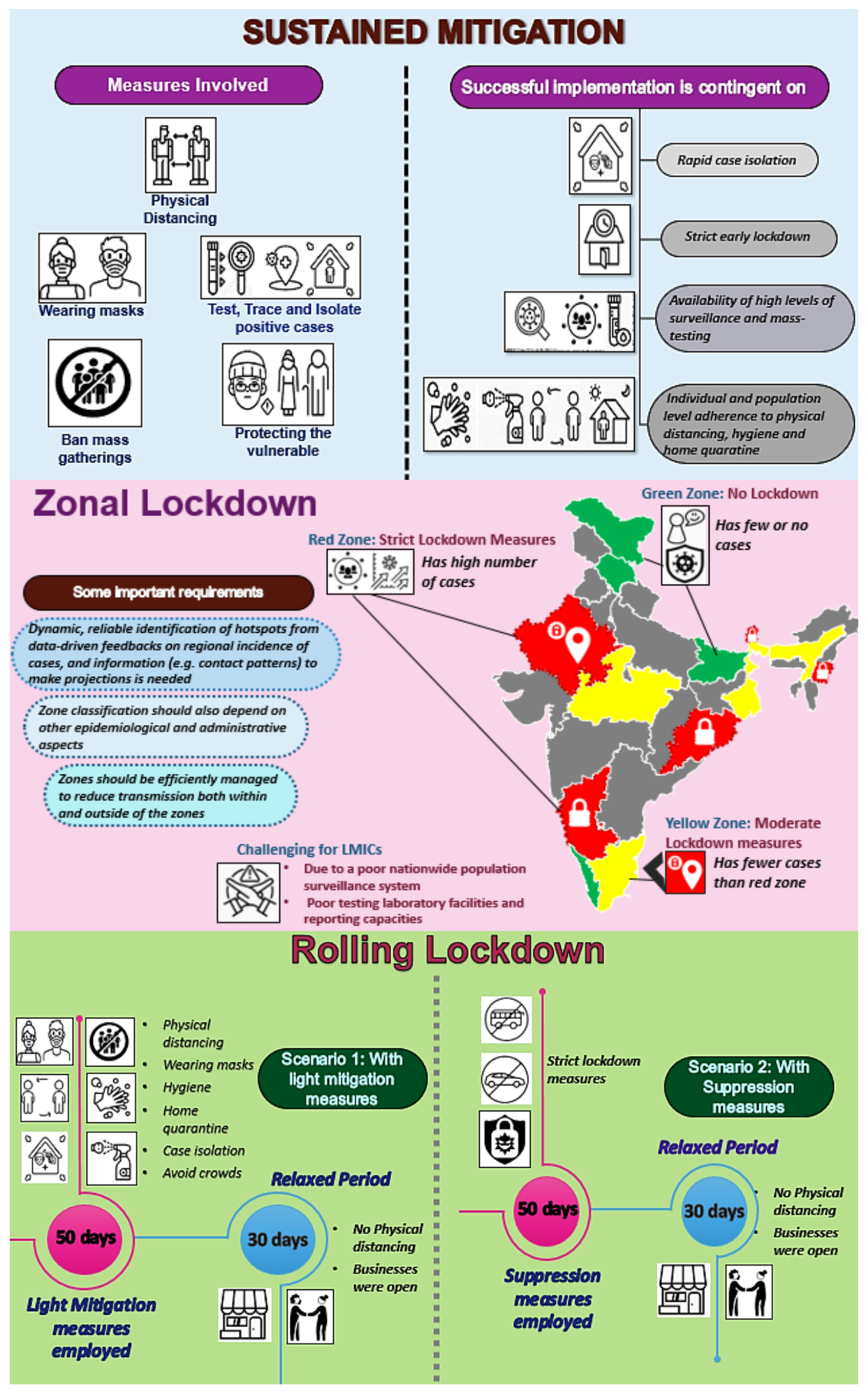




\section{REFERENCES}

1. Worldometer. COVID-19 CORONAVIRUS PANDEMIC. COVID-19 CORONAVIRUS PANDEMIC (2020). Available at: https://www.worldometers.info/coronavirus/. (Accessed: 8th June 2020)

2. Sterne, G. \& Sleptsova, E. Research Briefing | Global Coronavirus Watch: EMs weigh health vs economy. (2020).

3. Gopinath, G. The Great Lockdown: Worst Economic Downturn Since the Great Depression. IMFBlog Insights and Analysis on Economics and Finance (2020).

4. Banks, N. \& Hulme, D. Understanding the Impacts of Covid-19 on Livelihoods in Bangladesh: Findings from the PPRC-BIGD Rapid Response Survey. Glob. Dev. Inst. Blog (2020).

5. Nsubuga, P. et al. Chapter 53. Public Health Surveillance: A Tool for Targeting and Monitoring Interventions. in Disease Control Priorities in Developing Countries (2nd Edition) (2006). doi:10.1596/978-0-8213-6179-5/chpt-53

6. Walensky, R. P. \& del Rio, C. From Mitigation to Containment of the COVID-19 Pandemic. JAMA (2020). doi:10.1001/jama.2020.6572

7. Wilder-Smith, A. \& Freedman, D. O. Isolation, quarantine, social distancing and community containment: Pivotal role for old-style public health measures in the novel coronavirus (2019-nCoV) outbreak. J. Travel Med. 27, (2020).

8. Lau, H. et al. The positive impact of lockdown in Wuhan on containing the COVID-19 outbreak in China. J. Travel Med. 27, (2020).

9. Economist, T. Infections are rising fast in Bangladesh, India and Pakistan. The Economist (2020). Available at: https://www.economist.com/asia/2020/06/06/infections-are-risingfast-in-bangladesh-india-and-pakistan. (Accessed: 9th June 2020)

10. Commission, E. Coronavirus: a common approach for safe and efficient mobile tracing apps across the EU. (2020).

11. Steinbrook, R. Contact Tracing, Testing, and Control of COVID-19-Learning from Taiwan. JAMA Internal Medicine (2020). doi:10.1001/jamainternmed.2020.2072

12. United Nations. World Population Prospects 2019. Department of Economic and Social Affairs. World Population Prospects 2019. (2019).

13. Newagebd. Stigma around COVID-19 hampers the fight against it. www.newagebd.net (2020). Available at: https://www.newagebd.net/article/105701/stigma-around-covid19-hampers-the-fight-against-it. (Accessed: 9th June 2020)

14. Kapoor, G., Sriram, A., Joshi, J., Nandi, A. \& Laxminarayan, R. COVID-19 in India : Statewise estimates of current hospital beds, intensive care unit (ICU) beds and ventilators. (2020).

15. Riksbank, S. Monetary policy April 2020: The Riksbank is supporting an economy in crisis. 
(2020).

16. Oliu-Barton, M., Pradelski, B. \& Attia, L. Exit strategy: from self-confinement to green zones. EsadeEcPol Insight \#6 (2020).

17. Oliu-Barton, M. \& Pradelski, B. S. . Green zones: a mathematical proposal for how to exit from the COVID-19 lockdown. World Economic Forum (2020).

18. Hasell, J. et al. Statistics and Research Coronavirus (COVID-19) Testing. Our World in Data (2020). Available at: https://ourworldindata.org/coronavirus-testing\# .

19. MOHFW. Guideline for RT-PCR based pooled sampling for migrants/returnees from abroad/green zones. (2020).

20. MOHFW. Containment Plan Novel Coronavirus Disease 2019 (COVID 19) Version 2 (updated 16.05.2020). (2020).

21. Dutta, S. S. 15-30\% people in containment areas exposed to COVID-19: ICMR's serosurvey. The New Indian Express (2020). Available at:

https://www.newindianexpress.com/nation/2020/jun/08/15-30-people-in-containmentareas-exposed-to-covid-19-icmrs-serosurvey-2153893.html.

22. BBC. Coronavirus: Iran fears second wave after surge in cases. BBC News (2020). Available at: https://www.bbc.co.uk/news/world-middle-east-52903443. (Accessed: 8th June 2020)

23. Ferguson, N. M. et al. Impact of non-pharmaceutical interventions (NPIs) to reduce COVID-19 mortality and healthcare demand. Imperial College COVID-19 Response Team (2020). doi:10.25561/77482

24. Chowdhury, R. Rolling lockdowns could protect both economies and health in low-income countries. The Conversation (2020). Available at: https://theconversation.com/rollinglockdowns-could-protect-both-economies-and-health-in-low-income-countries-139054. (Accessed: 9th June 2020)

25. Chowdhury, R. et al. Dynamic interventions to control COVID-19 pandemic: a multivariate prediction modelling study comparing 16 worldwide countries. Eur. J. Epidemiol. 35, 389-399 (2020).

26. Kissler, S. M., Tedijanto, C., Goldstein, E., Grad, Y. H. \& Lipsitch, M. Projecting the transmission dynamics of SARS-CoV-2 through the postpandemic period. Science (80-. ). 368, 860-868 (2020).

27. Gabol, I. WHO says Pakistan meets no pre-requisites for easing restrictions, recommends 'intermittent lockdown'. Dawn (2020). Available at: https://www.dawn.com/news/1562406. 
